\title{
Robust pole placement using firefly algorithm
}

\author{
Moath Sababha, Mohamed Zohdy, Maged Kafafy \\ Electrical and Computer Engineering, Oakland University, Rochester, USA
}

\begin{tabular}{l}
\hline Article Info \\
\hline Article history: \\
Received Jul 12, 2018 \\
Revised Oct 18, 2018 \\
Accepted Nov 13, 2018 \\
\hline
\end{tabular}

Keywords:

Firefly algorithm

Multi-input-multi-output

(MIMO) systems

Proportional-integral derivative

(PID)

State feedback control

\begin{abstract}
In this paper, the new automatic tool that is based on the firefly algorithm whose purpose is optimization of pole location in the control of state feedback has been presented. The aim is satisfying specifications of performance like settling and rise time, steady state as well as overshoot error. Utilization of Firefly algorithm has demonstrated the benefits of controllers based on this kind of time domain over controllers based on the frequency domain like Proportional-Integral Derivative (PID). The presented method is more particular for the multi-input multi-output (MIMO) systems that have substantial state numbers. The simulation results indicated that the proposed method had superior performance in providing solution to the problems that involved stabilization of helicopter under the Rationalized Model of helicopter/ Moreover, it demonstrates the Firefly algorithm effectiveness with regards to, the state observer design and feedback controller and auto-tuning.
\end{abstract}

Copyright $(5) 2019$ Institute of Advanced Engineering and Science. All rights reserved.

\section{Corresponding Author:}

Moath Sababha,

Electrical and Computer Engineering,

Oakland University, Rochester, MI 48309 USA.

Email: msababha@ oakland.edu

\section{INTRODUCTION}

The classical control theory explains how feedback controllers satisfies the specifications of performance such as overshoot, rise time, steady state error and setting time. It also argues how root locus, Nyquist plot and Bode are approached by frequency domain and how simple controllers work such as Proportional-Integral-Derivative (PID) control. [1]-[3]. It is easy working in time domain in Multi-Input Multi-Output systems (MIMO) through the representation of the state space. It is possible because, with $p$ and $q$ inputs and outputs, the transfer functions $q \times p$ are essential in encoding of any equivalent information. The pole placement, commonly known as the state feedback control [4]-[6], achieve stability in closed-loop by involving the system pole relocation to the left half s-plane [7], [8].

Whereas compared to PID parameter turning procedure for pole placement is seem to be very straightforward, it is not clear as to how the closed-loop poles locations are related to the controller performance, specifically in transit state [9], [10]. Without counterparts that are sophisticated in turning methods that are frequency domain based, in the time domain, pole placement mainly is trial and error. In this state, several iterations of various pole location are attempted by control engineers until the desirable response is attained [11]-[13].

The availability of optimization techniques specifically the traditional ones is a challenge. Instead, the objectives of optimization exist only as a joint of transient parameters of performance (constants) where in this case there are incompatible gradients. This iterative search is highly dimensional despite being a potential search problem. Besides, feasibility is achieved only for problems of lower order. For instance, in linear system at $n$-state, routine for optimization will search in a $2 n$-dimensional hyperspace. Importance of doing so is to account for the imaginary and real parts in each pole. Furthermore, the search space is double by observer's design more efficiently. Such tasks with high dimensions cannot be handle by most optimization routines that are traditional [14]-[16]. 
For control design, the existing literature specifically on the previous work consider utilization of evolutionary algorithms. Scholars argue that the techniques do not require exact gradient information to for optimization to be obtained. In control engineering, to obtain the evolutionary algorithm overview, see [3], [4]. Particularly, pole placement in [11]-[13] came into being a problem of multi-objective optimization. However, the problem is solved by genetic algorithms (GAs).

This work can be compared more with [6] and [15], where GAs was utilized in MIMO systems that are nonlinear and for robust pole placement. Scientists recently utilized Particles Swarm Optimization (PSO) in tuning PID and PI controllers [15]-[17]. The work clearly demonstrated that the PSO is both a reliable and faster tool when it comes to control optimization. Therefore, it has better performance and provides better results compared to GA evolutionary algorithm.

The PID controller goal is determination of the parameters needed to meet the closed loop system specifications of performance as well as improving the robust performance of the control loop over various conditions of operation. In practical perspective, it is very hard to achieve simultaneously all the qualities desired. For instance, if adjustment is done to the PID controller with the goal of providing transient response to set point change, the resultant of this is sluggish response especially when put under conditions with disturbance. On the other hand, it the system is adjusted and made robust to disturbance which is done by choosing the PID controller's conservative values and may result to slow response of closed loop to a set change point. Thus, different methods of PID controllers tuning have been proposed. Conventional tuning method of PID control proposed in [12] is one of the well-known technique.

In practice, the method has effectively performed well. However, it has sometimes provided inadequate tuning in addition to its tendency to producing bigger overshoots. Thus, for effective application of this method, retuning is needed and should be applied prior to control industrial process. Additionally, in order to ensure that the method is enhanced especially enhancing the parameter tuning methods of traditional PID, several suggestions have been made in the approaches that can appropriately be employed to improve the tuning of PID. They include Generic algorithm, [18] and particle swarm optimization [19]. In the recent times, however, the advancement of computational methods has led to the proposal of optimization algorithm in order to tune the parameters of control in identifying the optimal Performa. Aside these efforts, literature exists on PID controllers, their enhancement and revolution of the PID controllers. The existence of this literature has been attributed to the fact that today, more than $50 \%$ of the industrial controllers are utilizing modified PID control or the PID control Schemes [20]. The widespread use and acceptance of the PDI controllers has mainly been as a result of robust performance and their simplicity in wide range of conditions of operations.

Thus, in enhancing the robustness of PID controllers, several literature exists. These previous work specifically uses the evolutionary algorithm which falls under the control design field. In control engineering [21]-[25] provided evolutionary algorithm overview. Furthermore, [26]-[28] used generic algorithm in the optimization of tanker autopilot control systems. Again, [19] utilized PSO in tuning of the linier giants of PID controller for Automatic Voltage Regulator (AVR) systems.

In another study, [29] utilized PSO to tune the parameters of proportional integral (PI) controller for double fed induction generators which is mainly drove by wind turbines. In addition, to swing up an acrobat with the assumption that there is limited torque, [30] came up with the GA-based method of control which proved to be an effective control method. In a more recent study conducted by [31], the evolutionary tuning techniques were employed specifically for a type 2-fuzzy logic controller. After this study, [32] conducted a study where a hybrid optimization algorithm was used, this involved a combination of pattern search based and PSO methods which were used in tuning of PI controller.

Further, classic example of inherent unstable system is inverted pendulum, and is an excellent test for testing and learning a number of control techniques. For instance, in stabilization of inverted pendulum system, [33] used sliding mode control using GA. The inverted pendulum linear state feedback controllers are mainly designed using the multi-objective uniform diversity GA and periodic multi-object PSO. In this case, an inverted pendulum system control mechanism is face-forward neural network novel fused controller and encoding of its parameters are done into real chromosomes values for GA [34].

In the light of the previous literature, the primary role of this paper is to optimize pole locations using Firefly algorithm in satisfying steady-state and transient performance. As Xin-She Yang proposed, when doing optimization using mathematics, optimization of firefly can be met holistically, and flashing behaviors of fireflies inspires it. When compared to other techniques of computerization intelligence such as genetic algorithm, neural networks as well as PSO, there is exceptional performance of firefly algorithm in optimization problem of high dimension.

The following paper section has been structured as follows. In Section 2, brief overview is given relating to time-dimension control design. It also discusses ways in which pole location hand tuning works to attain the transient and steady state performance. In Section 3, firefly algorithm is presented. The problem of 
pole placement is also formulated in this section using Firefly framework and offers details on design issues. Firefly optimized states feedback control is presented for helicopter stabilization case under the RHM in Section 4 and Section 5, respectively. The paper ends with a conclusion in Section 6.

\section{SYSTEM MODEL OF THE STATE FEEDBACK CONTROL}

The simple linear time-invariant (LTI) model is expressed as:

$$
\widehat{x}(\mathrm{t})=\mathbf{A x}(\mathrm{t})+\mathbf{B u}(\mathrm{t})
$$

where state vector is represented by $\mathbf{x}(\mathrm{t})$ and input vector is denoted by $\mathbf{u}(\mathrm{t})$. Then, the output vector $\boldsymbol{y}(\mathrm{t})$ is given by

$$
y(t)=C x(t)+D u(t)
$$

If the system is controllable, then the law of full state feedback that represents stable system of closed loop is given by

$$
\boldsymbol{u}(\mathrm{t})=-\mathbf{K x}(\mathrm{t})
$$

To that end, the controllability matrix of the linear system $\mathbf{R}$, can be expressed as

$$
\mathrm{R}=\left[\begin{array}{llll}
B & A B & \ldots & A^{n-1} B
\end{array}\right]
$$

where $\mathbf{R}$ is assumed to be full rank so that the system is controllable.

However, one can indirectly access the internal state $\mathbf{x}(\mathrm{t})$ of the system by using the separation principle techniques to design an observer to obtain from the output vector $\boldsymbol{y}(\mathrm{t})$ the input state vector $\mathbf{x}(\mathrm{t})$. Using the Luenberger observer, the output feedback model is given by:

$$
\begin{aligned}
& \overline{\bar{x}}(\mathrm{t})=\mathbf{A} \overline{\boldsymbol{x}}(\mathrm{t})+\mathbf{B u}(\mathrm{t})-\mathbf{L}(\overline{\boldsymbol{y}}(\mathrm{t})-\boldsymbol{y}(\mathrm{t})) \\
& \overline{\boldsymbol{y}}(\mathrm{t})=\mathbf{C} \overline{\boldsymbol{x}}(\mathrm{t})
\end{aligned}
$$

where it is assumed that there is observable system, that is observability matrix $Q=\left[\begin{array}{lll}\boldsymbol{C} & \boldsymbol{C} \boldsymbol{A} \ldots \boldsymbol{C} \boldsymbol{A}^{\boldsymbol{n}-1}\end{array}\right]^{T}$ contains a rank that is full. The output feedback techniques have been proved to work well in linear systems theory in the literature [12-25]. For more detailed derivation and designs refer to [2]. Also, the state feedback method has been used and exploited to solve the regulation problem and to track the reference output as in [20].

The challenge in system of state feedback control is to choose appropriate parameters for the feedback gains $\mathbf{K}$ and $\mathbf{L}$. These parameters rely on the closed loop poles. The advantage of using the state feedback method over the frequency-domain method is the pole placement process. In the state feedback method, the closed-loop system will give an error of zero-steady state when the poles are located in the s-plane left side. However, the lack of graphical tools will make it difficult to tune the rise time, settling time, and overshoot in the state feedback controller for a desired performance. To tackle this problem in [11-14], authors employ the linear quadratic regulator LQR to rely on optimal control. However, this method is still requiring manual tuning before a desirable response can be achieved.

\section{THE FIREFLY ALGORITHM}

In [22], the clarification on how the Firefly algorithm following the behavior of firefly is given. Firefly is an insect that for the most part delivers cadenced as well as short flashes that created by a bioluminescence procedure. The glimmering light capacity is pulling accomplices (correspondence) or drawing in potential prey and as a defensive cautioning toward the predator. Along these lines, this light power is the other fireflies' factor in advancing close to the other firefly.

At the separation, the light power is changed from the eyes of onlooker. It is protected to state that the light power is diminished as the distance increment. The light power likewise the impact of the air retains by the environment, in this way the force turns out to be less engaging as the separation increment. Firefly algorithm originally presented based on three idealize rules, 1) there is an attraction of fireflies towards one 
another without considering the gender. 2) There is correlative engaging quality of the fireflies with fireflies' splendor. Consequently, firefly that is less appealing tends to push ahead towards the firefly that is more alluring. 3) Fireflies shine relies upon the cost function [22].

\subsection{Firefly algorithm structure}

In firefly algorithm, there exist two essential factors, these are the light intensity appeal and force. Firefly is pulled in toward the other firefly containing blaze that is brighter than its braze. The engaging quality is depending on the light power.

The light intensity accordingly attractiveness is inversely relative with the particular distance $\boldsymbol{r}$ from the light source. In this manner, the light and engaging quality is diminishing as the distance increment. One can express it as follows:

$$
I(r)=I_{0} e^{-\gamma r^{2}}
$$

where,

$I \quad=$ Stands for light intensity,

$I_{0}=$ Stands for light intensity, at original or initial light intensity

$\gamma=$ Stands for the coefficient of the light absorption

$r \quad=$ Represents the distance between $i$ and $j$ firefly

Considering that attractiveness is proportional to the seen by another fireflies' light intensity, then the attractiveness $(\beta)$ is given as

$$
\beta=\beta_{0} e^{-\gamma r^{2}}
$$

where $\beta_{0}$ represents the attractiveness at zero distance $(r=0)$. The distance $r$ between two fireflies can be defined based on the Cartesian distance as follows:

$$
r_{i j}=\left|x_{i}-x_{j}\right|=\sqrt{\sum_{k=1}^{d}\left(x_{i, k}-x_{j, k}\right)^{2}}
$$

Firefly $i$ is attracted toward the more attractive firefly $j$, the updated movement is expressed as follows:

$$
\Delta x_{i}=\beta_{0} e^{-\gamma r_{i j}^{2}}\left(x_{j}^{t}-x_{i}^{t}\right)+\alpha \varepsilon_{i}, \quad x_{i}^{t+1}+\Delta x_{i}
$$

Where attraction is done by $\beta_{0}$, limitation is $\gamma$ when there is tendency of too large value or tends to be zero. There is constant brightness and attractiveness $\beta=\beta_{0}$ if zero is being approached $(\gamma \rightarrow 0)$, by $\gamma$. This means in short that it is possible to see in any position the firefly, easy in completing the global search. If the $\gamma$ is very large or is nearing infinity $(\gamma \rightarrow \infty)$, then the brightness and attractiveness on the other hand decreases. There is thus a random movement of firefly. The two asymptotic behavior as used to conduct the firefly algorithm implementation. As for randomized parameter is $\alpha$, the second the term is used for randomization. It is possible to replace the $\varepsilon_{i}$ by $\mathrm{ran}-1 / 2$ ran as random number and is generated from 1 to 0 . Please refer to Algorithm 1.

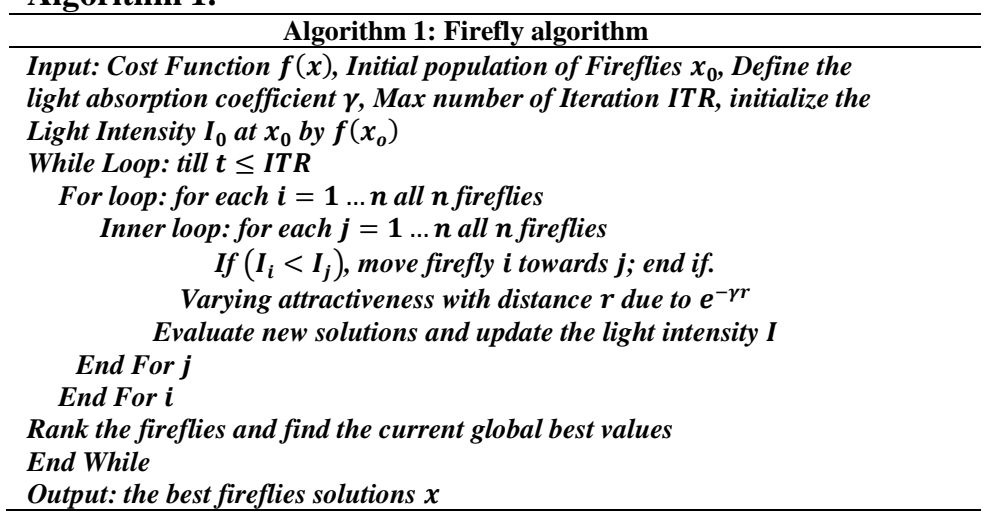




\section{OBJECTIVE FUNCTION}

In order to address the problem of optimal pole placement based on Firefly algorithm, we place the pole locations as firefly's positions. However, one can represent the dimensions of the firefly's search space by assuming the poles to be complex conjugates as follows [15]:

$$
\mathrm{x}_{\mathrm{i}}=\mathrm{a} \pm \mathrm{jb}
$$

where each pair of poles can be represented by a set values of a and $b$ from (11).

In the Firefly algorithm, the objective function is optimized without using any gradient methods.However, we use the objective function that evaluates the pole locations in terms of steady state error, settling time, Overshoot rise time and maximum input limit. So, the desired objective function is expressed as the sum of all individual values including steady state error, settling time, Overshoot rise time and maximum input limit.

\section{SIMULATION RESULTS AND ANALYSIS}

In this section, The proposed control strategy is validated by simulation. Simulation is executed in the Matlab/Simulink environment. In this paper, we chose a helicopter stabilization design as a proof of concept problem in demonstrating the Firefly-based state feedback design. The Rationalized Helicopter Model (RHM) [17], [26]-[28] is a well-studied nonlinear dynamical model of single rotor helicopters. Modeled after the Westland Lynx helicopters, the RHM accounts for a four-blade semi-rigid main rotor and rigid body. The equations governing the helicopter motions are complex. Additionally, the open loop dynamics are unstable throughout the flight envelope, exhibiting a highly cross-coupled and nonlinear response. The state vector $\mathbf{x}(\mathrm{t})$ of the dynamical model contains eight states [19], i.e. pitch attitude $(\theta)$, roll attitude $(\varnothing)$, roll rate $(p)$, pitch rate $(q)$, yaw rate $(\xi)$, forward velocity $\left(v_{x}\right)$, lateral velocity $\left(v_{y}\right)$ and vertical velocity $\left(v_{z}\right)$. Moreover, the output state vector $\mathbf{y}(\mathrm{t})$ contains four controlled signals, i.e. heave velocity $\left(H_{t}\right)$, pitch attitude $(\theta)$, roll attitude $(\emptyset)$, and heading rate $\left(\psi_{t}\right)$ and two additional body-axis measurements (roll rate $(p)$, pitch rate $(q))$. Also, there are four blade angles serve as the inputs to the helicopter as follows:
a. $u_{1}$ - main rotor collective
b. $u_{2}$ - longitudinal cyclic
c. $u_{3}$ - lateral cyclic
d. $u_{4}$ - tail rotor collective

The MATLAB script of the $\boldsymbol{A}, \boldsymbol{B}, \boldsymbol{C}$ matrices of the RHM model can be obtained in [19].

In summary, the main rotor collective input controls the lift by rotating the rotor blades. The longitudinal and lateral cyclic inputs control the longitudinal and lateral motions by varying the blade angles. The tail rotor balances the torque generated by the primary rotor to prevent the aircraft from spinning and give it a desired lateral motion. This model assumes that the dynamics get decoupled. However, in reality, they have an extreme coupling, resulting in non-minimum phase features in particular operation points. More discussion on dynamics is available in [17], [19].

In this paper, we employed the Firefly algorithm to perform robust control design to reduce the effects of atmospheric turbulence in the RHM model. The paper will focus on the task of optimization for transient state performance. Moreover, it will then underline the impressive dimensional search capabilities of the algorithm for the eight-state RHM model. It will also discuss the Firefly algorithm searches for optimal output feedback-controller design in R16.

Consider the scenario of a helicopter in motion in some non-zero initial state. The objective of control is to ensure system stabilization as if in the original state. In other words, it aims to reach a state of equilibrium where the vector sum of all forces and all moments is equal to zero. Since the objective of control is not tracking step reference, the paper has not included other transient performance metrics such as overshoot and rise time.

After the construction of the controllability and observation matrices, it is procedural to ensure that the plant is both controllable and observable. A series of hand-tuned simulations are conducted first within a 5 -second duration and a time step of 0.01. Alternatively, the simulation identifies pole locations to satisfy a steady state error requirement, then the settling time, and lastly maximum input. After ten iterations in approximately five minutes, the response in Figure 1 results. When the maximum input still does not fall under the desired level, the reaction in Figure 2 ensues. Further simulations either increase $u(t)$ or violate the first two constraints. 


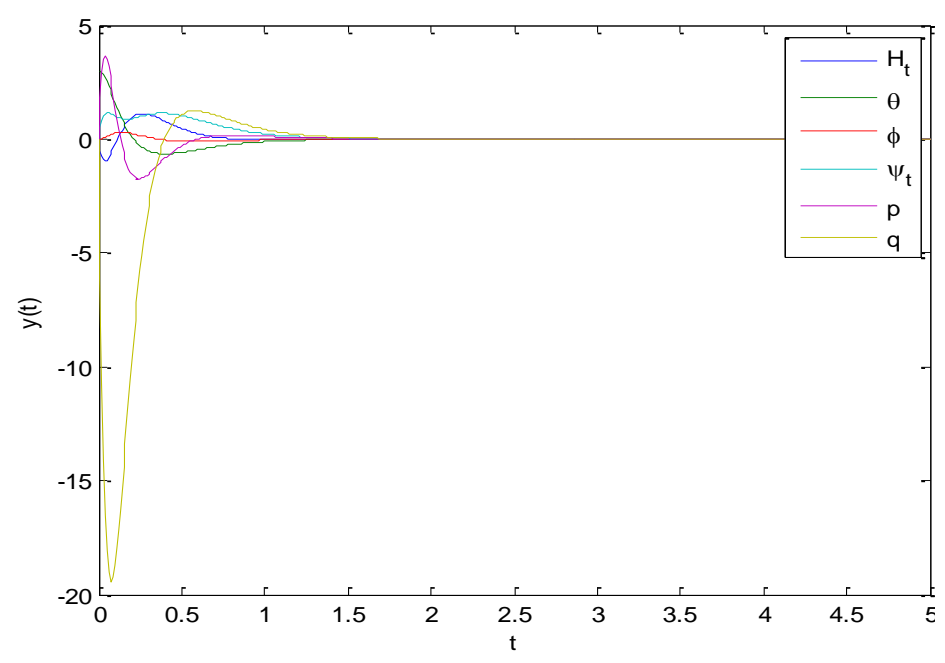

Figure 1. Hand-tuned pole placement simulation showing the six outputs stabilizing to zero in 2 seconds

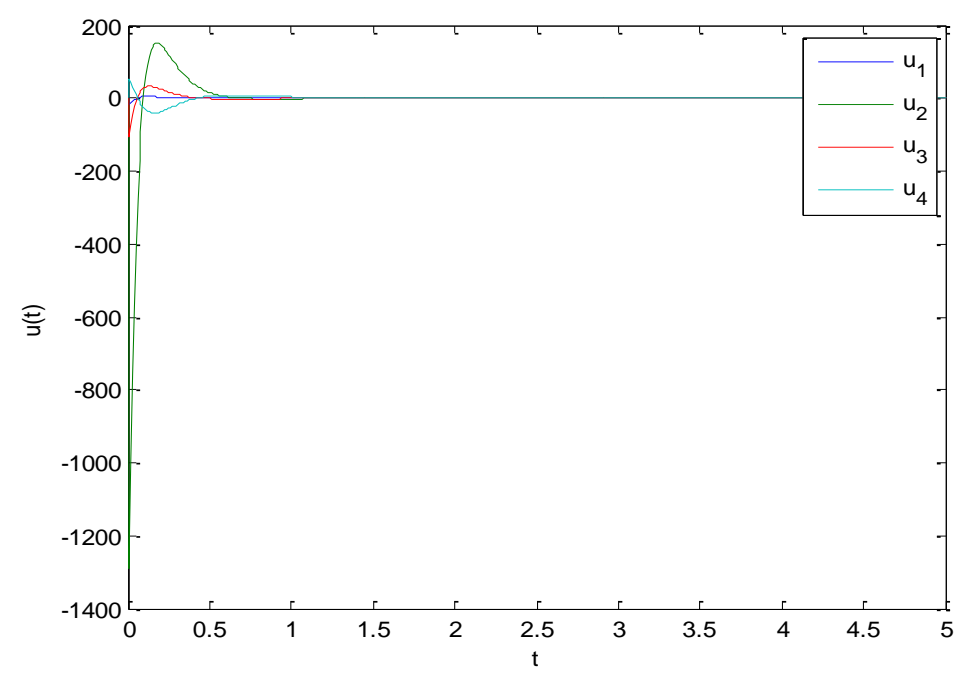

Figure 2. Hand-tuned control inputs corresponding to Figure 1 with a maximum value of 120.5 (u2)

The Fireflies positions exist in a $2 \times 6 \times 8$ matrices, which accounts for two axes (imaginary and real). It also comprises of eight fireflies and six complex-conjugate poles ( 3 for the plant, 3 for the observer). Every parameter available in the multiple inputs and outputs faces an equal penalty when calculating the objective function. Illustrations on the plots corresponding to the Firefly-tuned simulations are available in Figure 3 and Figure 4.

Auto-tuned design based on Firefly has been simulated using Matlab software on Intel Core i5 2.4 $\mathrm{GHz}$ computer with a memory of $4 \mathrm{~GB}$. The stimulation takes approximately 9 seconds (or less time than a single hand-tuned iteration) to complete.

Various other combinations of Firefly parameters are simulated to improve system response even further. We increased the number of Fireflies and the maximum number of iterations as shown in Figure 5 and Figure 6. It obviously yields slight variations in the output performance. Extending the variation in Firefly parameters shows tradeoffs between the settling time and the maximum input. Nonetheless, the parameters used in the initial set seem to give a desirable performance within the shortest computation time. 


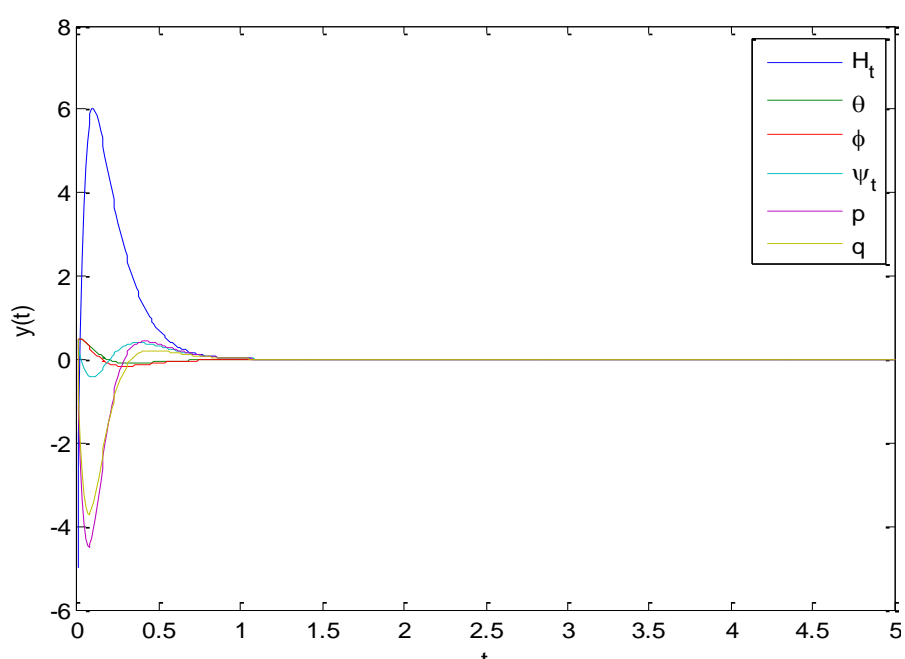

Figure 3. FireFly-based method simulation showing that the six outputs stabilize to zero in 1.3 seconds

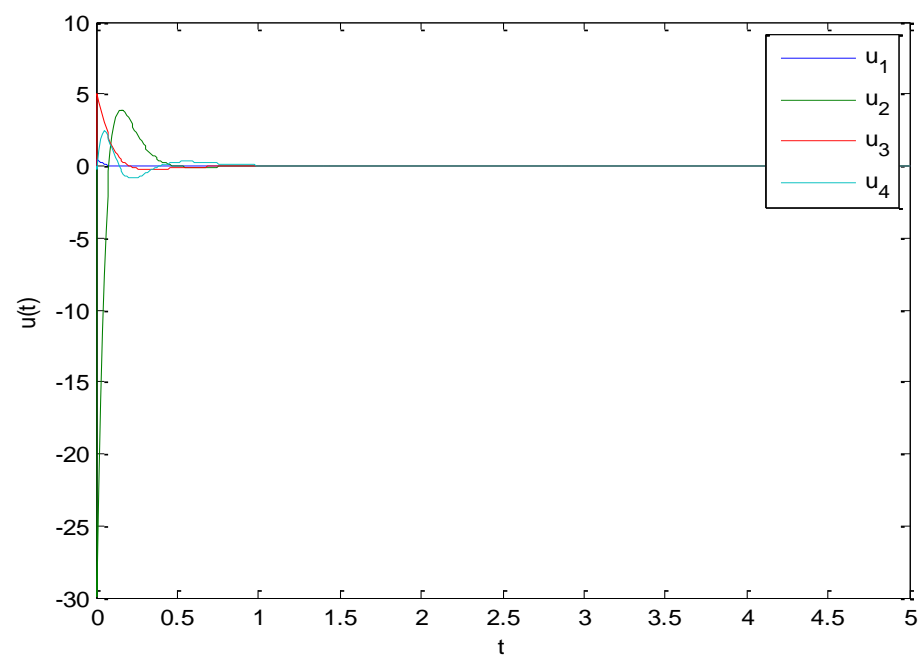

Figure 4. FireFly-based control inputs corresponding to Figure 3

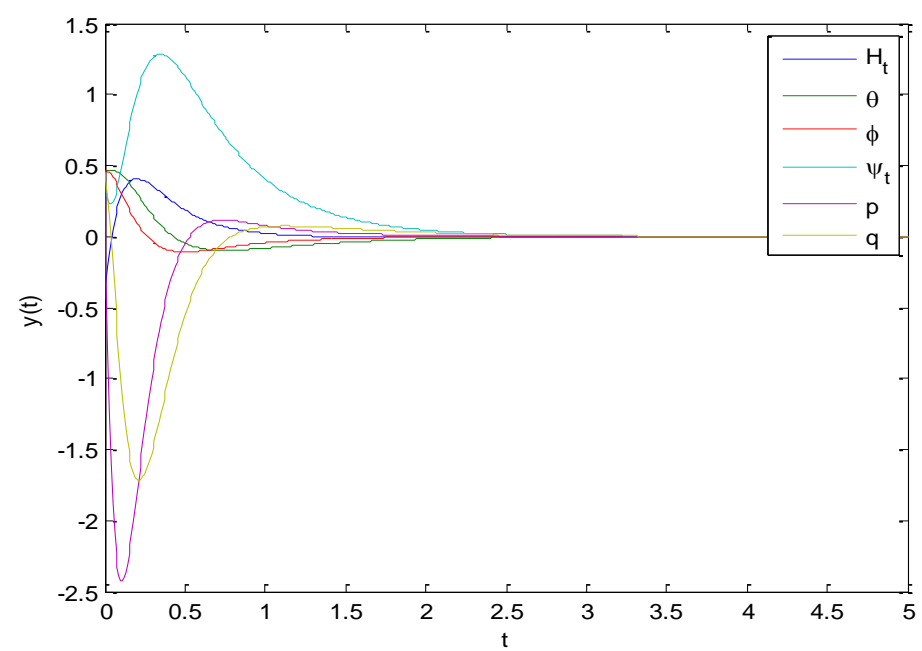

Figure 5. FireFly-based method (12 Fireflies) simulation showing that the six outputs stabilize to zero in 2.5 seconds 


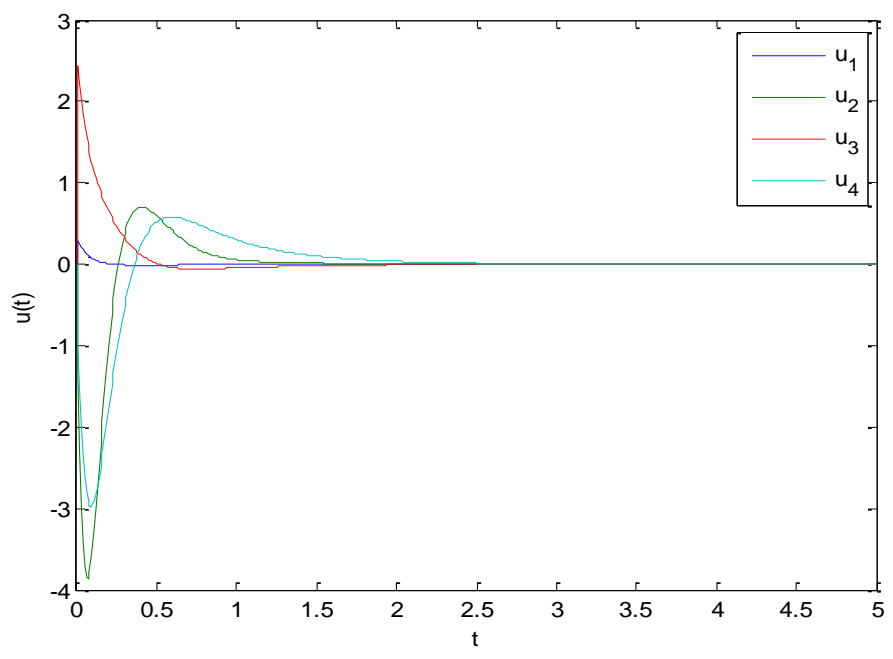

Figure 6. FireFly-based control inputs corresponding to Figure 5

\section{CONCLUSION}

This paper employs the Firefly method to obtain the optimal pole locations in state feedback control. It also aimed to satisfy transient and steady-state performance requirements such as rise time, overshoot, settling time, and steady-state error. It presented proof of a concept problem involving the stabilization of a helicopter. It also delivered results of Firefly algorithm auto-tuning and supported them with computer simulations.

\section{REFERENCES}

[1] W. Brogan, Modern Control Theory, 3rd ed. Upper Saddle River, NJ: Prentice Hall, 1991.

[2] Bouabdallah, S., "Design and Control of Quadrotors with Application to Autonomous Flying," Ph.D. dissertation, École Polytechnique Federale De Lausanne, 2007.

[3] S. Mohammad Mirzaei; Mohammad H. Moattar, "Optimized PID Controller with Bacterial Foraging Algorithm," International Journal of Electrical and Computer Engineering (IJECE), Vol. 5, No. 6, December 2015 ,

pp. 1372-1380.

[4] N. R.RajuandP. L.Reddy,"Optimal Tuning of Fractional Order PID Controller for Automatic Voltage Regulator System through Genetic Algorithm," International Journal of Engineering and Technology, vol/issue:8(2), pp. 922-927, 2016.

[5] Z. Albataineh, F. Salem, "Robust blind multiuser detection algorithm using fourth-order cumulant matrices" Circuits, Systems, and Signal Processing 34 (8), 2577-2595, 2015.

[6] Albataineh, Z. Telecommun Syst (2018) 68: 573. https://doi.org/10.1007/s11235-017-0410-4

[7] Z. Albataineh, "Blind Decoding of Massive MIMO Uplink Systems Based on the Higher Order Cumulants" Wireless Personal Communications, https://doi.org/10.1007/s11277-018-5883-2, 2018.

[8] Albataineh, Z. \& Salem, F.M. Circuits Syst Signal Process (2017) 36: 3320. https://doi.org/10.1007/s00034-0160459-4

[9] N. Ramesh Raju. and P. Linga Reddy, "Robustness Study of Fractional Order PID Controller Optimized by Particle Swarm Optimization in AVR System," International Journal of Electrical and Computer Engineering (IJECE), Vol. 6, No. 5, October 2016, pp. 2033-2040.

[10] P. Fleming and R. Purshouse, "Evolutionary algorithms in control systems engineering: a survey," Control Engineering Practice, vol. 10, no. 11, pp. 1223-1241, 2002.

[11] C. Fonseca and P. Fleming, "Multiobjective optimal controller design with genetic algorithms," International Conference on Control, vol. 1, pp. 745-749, March 1994.

[12] G. Sanchez, M. Villasana, and M. Strefezza, "Multi-objective pole placement with evolutionary algorithms," Lecture Notes in Computer Science, vol. 4403, p. 417, 2007.

[13] J. Ouyang and W. Qu, "Robust pole placement using genetic algorithms," in International Conference on Machine Learning and Cybernetics, vol. 2, 2002.

[14] Bouabdallah, S.; Siegwart, R., "Full control of a quadrotor," Intelligent Robots and Systems, 2007. IROS 2007. IEEE/RSJ International Conference on, vol., no., pp.153,158, Oct. 29 2007-Nov. 22007.

[15] J. Wang, B. T. Brackett and R. G. Harley, "Particle Swarm-Assisted State Feedback Control: From Pole Selection to State Estimation”, 2009 American Control Conference, June 10-12, 2009 
[16] W. Qiao, G. Venayagamoorthy, and R. Harley, "Design of optimal PI controllers for doubly fed induction generators driven by wind turbines using particle swarm optimization," 2006 International Joint Conference on Neural Networks, pp. 1982-1987, 0-0 2006.

[17] D. Mandal, S. P. Ghoshal, and A. K. Bhattacharjee, "A Novel Particle Swarm Optimization Based Optimal Design of Three-Ring Concentric Circular Antenna Array," IEEE International Conference on Advances in Computing, Control, and Telecommunication Technologies, 2009. (ACT'09), pp. 385-389, 2009.

[18] Kumbharana, S. and Pandey, G, (2013), "Solving Travelling Salesman Problem using Firefly Algorithm," International Journal for Research in Science \& Advanced Technologies, 2(2), pp. 53-57.

[19] Farook, S. and Raju, P, (2013), "Evolutionary Hybrid Genetic-Firefly Algorithm for Global Optimization. IJCEM," International Journal of Computational Engineering \& Management, 16(3), pp. 37-45.

[20] A. E. Bryson and Y.-C. Ho, "Applied Optimal Control: Optimization, Estimation, and Control. Washington," DC: Hemisphere Publishing Corporation, 1975.

[21] J. Kennedy and R. C. Eberhart, Swarm Intelligence. San Francisco, CA, USA: Morgan Kaufmann Publishers Inc., 2001.

[22] Yang, X. (2010). Nature-Inspired Metaheuristic Algorithms. 2nd ed. Frome: Luniver Press.

[23] J. Butcher, Numerical methods for ordinary differential equations. Wiley, 2003.

[24] U. M. Ascher and L. R. Petzold, "Computer Methods for Ordinary Differential Equations and DifferentialAlgebraic Equations," SIAM: Society for Industrial and Applied Mathematics, 1998.

[25] J. Kautsky, "Robust pole assignment in linear state feedback," International Journal of Control, vol. 41, no. 5, pp. 1129-1155, May 1986.

[26] G. Padfield, "A theoretical model of helicopter flight mechanics for application to piloted simulation," $R A E$ Technical Report, vol. 81048, 1981.

[27] S. Skogestad and I. Postlethwaite, Multivariable Feedback Control: Analysis and Design. Wiley, 2005.

[28] S. Skogestad. (2008, September) Rationalized helicopter model. [Online]. Available: http://www.nt.ntnu.no/users/skoge/book/2nd edition/matlab.html

[29] Z. Albataineh, F. Salem, "New Blind Multiuser Detection in DS-CDMA Using H-DE and ICA Algorithms," International Conference on 2013 4th Intelligent Systems Modelling \& Simulation (ISMS), pp. 569-574, Jan. 2013.

[30] Z. Albataineh, F. Salem and J. I. Ababneh, "Linear phase FIR Low Pass Filter Design Using Hybrid Differential Evolution", International Journal of Research in Wireless Systems (IJRWS), Vol. 1, Issue 2, pp. 43-49, December 2012.

[31] P. Cominos and N. Munro, "PID controllers: recent tuning methods and design to specification," IEE Proceedings on Control Theory and Applications, vol. 149, no. 1, pp. 46-53, Jan 2002.

[32] Yu, S., Yang, S. and Su, S, (2013), "Self-Adaptive Step Firefly Algorithm," Journal of Applied Mathematics, pp. 1-8.

[33] Piasecki, J.S.; Zohdy, M.A, "Hybrid Dynamic System Control of Sequential Motion in Biped Robots," ACSE Journal, Volume 14, Issue 1, ISSN 1687-4811, August 2014

[34] Andalib Sahnehsaraei M, Mahmoodabadi MJ and Bagheri A, (2013), "Pareto optimum control of a 2-DOF inverted pendulum using approximate feedback linearization and sliding mode control," Transactions of the Institute of Measurement and Control doi: 0142331213504946.

\section{BIOGRAPHIES OF AUTHORS}

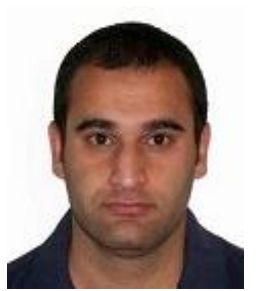

Moath Sababha was born in Irbid, Jordan in 1987. He received his B. Sc. degree in Electrical Engineering from Jordan University of Sc. and Tech. JUST, Irbid, Jordan in 2010. He received his M. Sc. degree in Electrical Engineering from Oakland University, Rochester, Michigan, U.S.A in 2013. He is currently a Ph. D. candidate in Electrical and Computer Engineering at Oakland University, Rochester, Michigan, U.S.A. His research interests are in the areas of digital signal processing, nonlinear estimation and prediction, fuzzy logic and decision making.

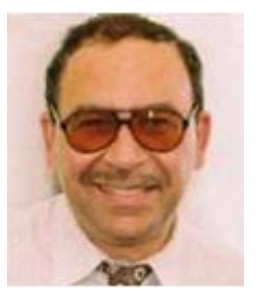

Mohamed A. Zohdy was born in Caro, Egypt. He received B.Sc. degree in Electrical Engineering, Cairo University, Egypt in 1968 and he received his M. Sc. and Ph. D. degrees in Electrical Engineering from University of Waterloo, Canada in 1974, and 1977 respectively. He worked in various industries: dowty, iron and steel, and spar. He is currently a Professor at Oakland University, Rochester Hills, Michigan, U.S.A. His research interests are in areas of control, estimation, communication, neural networks, fuzzy logic and hybrid systems. 